\title{
高熵化设计：稀土硅酸盐材料关键性能优化新策略
}

\author{
孙鲁超 ${ }^{1}$ ，任孝旻 ${ }^{1,2}$, 杜铁锋 ${ }^{1}$ ，罗颐秀 ${ }^{1}$, 张 洁 $^{1}$ ，王京阳 ${ }^{1}$
}

(1. 中国科学院金属研究所, 陶瓷及复合材料研究部, 沈阳 $110016 ; 2$. 中国科学技术大学, 材料科学与工程学院, 合肥 230026)

摘 要: 环境障涂层是先进航空发动机高温结构部件用碳化硅纤维增强碳化硅 $\left(\mathrm{SiC}_{\mathrm{f}} / \mathrm{SiC}\right)$ 陶瓷基复合材料的关键防 护。稀土硅酸盐具有低热膨胀系数、优良的抗水氧/CMAS 腐蚀性能以及与硅基陶瓷良好的化学相容性, 是目前国 际公认的优选环境障涂层体系。常规含单一稀土元素的稀土硅酸盐环境障涂层材料, 存在热应力失配、高温相转变 和耐腐蚀性能差等问题, 尚无法完全满足极端燃气环境中的长寿命服役要求。本综述介绍了为解决稀土硅酸盐环境 障涂层的关键性能局限, 利用高熵化合物设计方法, 针对稀土硅酸盐热学性能(热膨胀系数和热导率)调控、耐 CMAS 腐蚀性能提升和相结构稳定性优化方面获得的新进展。这些研究进展为稀土硅酸盐材料的创新设计提供了 新思路, 为其作为下一代环境障涂层的性能突破提供了支撑。

关 键 词: 高熵化设计; 高熵陶瓷; 稀土硅酸盐; 环境障涂层; 综述

中图分类号: TQ174 文献标识码: A

\section{High Entropy Engineering: New Strategy for the Critical Property Optimizations of Rare Earth Silicates}

\author{
SUN Luchao ${ }^{1}$, REN Xiaomin ${ }^{1,2}$, DU Tiefeng ${ }^{1}$, LUO Yixiu ${ }^{1}$, ZHANG Jie $^{1}$, WANG Jingyang ${ }^{1}$ \\ (1. Advanced Ceramics and Composites Division, Institute of Metal Research, Chinese Academy of Sciences, Shenyang 110016, \\ China; 2. School of Materials Science and Engineering, University of Science and Technology of China, Hefei 230026, China)
}

\begin{abstract}
Environmental barrier coatings (EBCs) have been developed to improve the durability of $\mathrm{SiC}_{\mathrm{f}} / \mathrm{SiC} \mathrm{CMC}$ components against harsh combustion environment. Among the most promising EBC candidates, rare-earth (RE) silicates attract attentions for their low thermal expansion coefficient, excellent high temperature water vapor and CMAS corrosion resistance, and good thermal and chemical compatibility with silicon-based ceramics and composites. Herein, we reviewed the optimizations of critical properties of rare-earth silicates through strategic high entropy design to modify the current performance deficiencies of rare-earth silicates like thermal properties (coefficient of thermal expansion and thermal conductivity), CMAS corrosion resistance and high temperature phase stability. The present advancements demonstrate the merits of high entropy engineering for advanced EBCs for the improvement of crucial properties in engine applications.
\end{abstract}

收稿日期: 2020-10-27；收到修改稿日期：2020-12-14；网络出版日期：2020-12-21

基金项目: 航空发动机及燃气轮机重大专项基础研究项目(2017-VI-0020-0093); 国家自然科学基金(51772302); 中科院国 际伙伴计划对外合作重点项目(174321KYSB20180008); 辽宁省自然基金(2020-MS-006)

National Science and Technology Major Project (2017-VI-0020-0093); National Natural Science Foundation of China (51772302); International Cooperation Key Program (174321KYSB20180008); Natural Science Foundation of Liaoning Province (2020-MS-006)

作者简介：孙鲁超(1984-), 男, 副研究员. E-mail: 1csun@imr.ac.cn SUN Luchao(1984-), male, associate professor. E-mail: 1csun@imr.ac.cn

通信作者：王京阳，研究员. E-mail: jywang@imr.ac.cn WANG Jingyang, professor. E-mail: jywang@imr.ac.cn 
Key words: high entropy engineering; high entropy ceramics; rare earth silicate; environmental barrier coatings; review

高熵合金(High Entropy Alloys, HEAs)通常是指 由 5 种及以上元素按等原子比或近等原子比合金化 形成的固溶体材料 ${ }^{[1-5]}$ 。熵是一个热力学系统混乱程 度的量度, 体系混乱程度越高, 熵越大。相比于传统 材料, 高熵合金通常具有更优的结构稳定性和综合 力学性能, 部分还表现出优异的物理 (电学、磁学) 和化学(抗腐蚀和催化)等性能, 是一类应用潜力巨 大的新型材料 ${ }^{[6-12]}$ 。目前关于高熵合金优异性能的 来源主要有四种解释: 热力学的高熵效应、动力学 的扩散迟滞效应、结构的晶格畸变效应以及性能提 升的鸡尾酒效应 ${ }^{[13-14]}$ 。

2015 年, Rost 等 ${ }^{[15]}$ 首次报导了一种岩盐结构的 (MgNiCoCuZn)O 高熵陶瓷材料, 将“高熵”概念从合 金扩展到陶瓷材料领域。随后, 越来越多的高熵陶 瓷体系, 如高熵氧化物 ${ }^{[16-18]}$ 、嗍化物 ${ }^{[19]}$ 、碳化物 ${ }^{[20-28]}$ 、 氮化物 ${ }^{[29]}$ 、硅化物 ${ }^{[30]}$ 、磷酸盐 ${ }^{[31]}$ 和锆酸盐 ${ }^{[32]}$ 等被广 泛报道。目前高摘陶瓷还没有绝对的定义, 其概念 由高熵合金发展而来, 通常定义为含有 5 种以上组 元的固溶体陶瓷材料, 或根据 $S_{\mathrm{mix}} \geqslant 1.61 R\left(S_{\mathrm{mix}}\right.$ 为混 合熵, $R$ 为理想气体常数)来定义。也有部分文献把

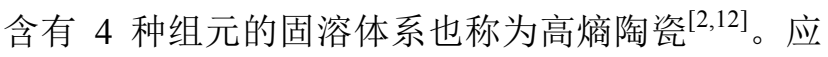
该说高熵陶瓷的概念还在不断完善与发展中, 但是 与传统陶瓷材料相比, 已知的高熵陶瓷在成分、结构 或性能上表现出明显的差异, 突破了简单组元成分 的设计局限, 为新材料体系研发和关键性能优化提 供了更广阔的思路和指导, 基于高熵化策略来探索陶 瓷材料的性能优化已经成为本领域的一大热点。

以碳化硅纤维增强碳化硅 $\left(\mathrm{SiC}_{\mathrm{f}} / \mathrm{SiC}\right)$ 为代表的 陶瓷基复合材料具有低密度、高温力学性能及抗氧 化性能优异等特点, 应用于航空发动机的高温结构 部件时, 可提高浴轮前温度约 $400 \sim 500{ }^{\circ} \mathrm{C}$, 大幅减 重(减重 $1 / 3 \sim 2 / 3$ ), 减少对部件冷却结构设计的需求, 降低了部件的制造难度, 是未来发动机热端结构部 件的首选材料。 $\mathrm{SiC}_{\mathrm{f}} / \mathrm{SiC}$ 陶瓷基复合材料在干燥氧 化气氛中, 表面能形成致密、抗氧化的 $\mathrm{SiO}_{2}$ 保护层。 在航空发动机燃气环境中, $\mathrm{SiO}_{2}$ 保护层会与水蒸气 反应生成挥发性 $\mathrm{Si}(\mathrm{OH})_{4}$, 造成氧化膜失效及复合 材料的挥发与损伤, 降低结构部件的力学性能与服 役可靠性。因此, 必须在部件表面涂覆环境障涂层 (Environmental Barrier Coating, EBC), 阻止或减缓 发动机燃气环境对陶瓷基复合材料高温结构部件的 腐蚀与损伤。可以说, 高稳定性环境障涂层是 $\mathrm{SiC}_{\mathrm{f}} / \mathrm{SiC}$ 复合材料应用于高推重比航空发动机的关
键技术。 $\mathrm{EBC}$ 材料的发展经历过三个阶段 ${ }^{[33]}$ : 第一 代和第二代 $\mathrm{EBC}$ 以莫来石 $\left(3 \mathrm{Al}_{2} \mathrm{O}_{3} \cdot 2 \mathrm{SiO}_{2}\right)$ 和钡锶铝 硅酸盐(BSAS)为主体材料, 由于高温下化学稳定性 和相容性差等原因, 它们只能应用于 $1300{ }^{\circ} \mathrm{C}$ 以下 的服役环境; 第三代及之后的 $\mathrm{EBC}$ 以满足更极端服 役环境 $\left(>1300{ }^{\circ} \mathrm{C}\right)$ 和更长服役时间 $(>500 \mathrm{~h})$ 为首要目 标, 稀土硅酸盐与 $\mathrm{SiC}_{\mathrm{f}} / \mathrm{SiC}$ 陶瓷基复合材料基体的 热膨胀系数和化学相容性(如稀土双硅酸盐)匹配性 好, 抗水氧和 CMAS 腐蚀性能与相稳定性(如稀土 单硅酸盐)优良, 有望在 $1300{ }^{\circ} \mathrm{C}$ 或者更高温度的燃 气环境中长寿命服役, 是目前国际公认的 $\mathrm{EBC}$ 优选 体系 ${ }^{[34]}$ 。

\section{EBC 用稀土硅酸盐研究现状与性能 局限}

新一代 $\mathrm{SiC}_{\mathrm{f}} / \mathrm{SiC}$ 复合材料用 $\mathrm{EBC}$ 对材料的综合 性能要求极为严苛, 要求材料同时具备优异的高温 稳定性、抗热震性、高损伤容限、抗高温氧化和腐 蚀性能, 以及与 $\mathrm{SiC}_{\mathrm{f}} / \mathrm{SiC}$ 陶瓷基复合材料良好的热 膨胀匹配性和化学相容性。稀土硅酸盐材料家族庞 大, 体系丰富, 其中稀土单硅酸盐 $\left(\mathrm{RE}_{2} \mathrm{SiO}_{5}\right)$ 和双硅 酸盐 $\left(\mathrm{RE}_{2} \mathrm{Si}_{2} \mathrm{O}_{7}, \mathrm{RE}\right.$ 表示稀土元素)是最优的 $\mathrm{EBC}$ 候 选材料。稀土单硅酸盐 $\mathrm{RE}_{2} \mathrm{SiO}_{5}$ 具有两种多型相, 即 稀土离子半径较大的稀土元素( $\mathrm{La} \sim \mathrm{Gd}$ )形成的 X1 相, 稀土离子半径较小的元素 $(\mathrm{Tb} \sim \mathrm{Lu}$ )形成的 $\mathrm{X} 2$ 相。 $\mathrm{RE}_{2} \mathrm{SiO}_{5}$ 具有热导率低、相稳定性好、耐水蒸气和 CMAS 腐蚀性能好的优点, 但是与 $\mathrm{Si}$ 或 $\mathrm{SiO}_{2}$ 化学相 容性差, 且热膨胀系数较大, 达到(6.94 8.84) $\times 10^{-6} \mathrm{~K}^{-1}$, 与 $\mathrm{SiC}_{\mathrm{f}} / \mathrm{SiC}$ 的热膨胀系数 $\left((3.5 \sim 5.5) \times 10^{-6} \mathrm{~K}^{-1}\right)$ 不匹 配 ${ }^{[34-35]}$ 。稀土双硅酸盐 $\mathrm{RE}_{2} \mathrm{Si}_{2} \mathrm{O}_{7}$ 目前已发现 7 种多 型结构, 除 $\mathrm{Sc}_{2} \mathrm{Si}_{2} \mathrm{O}_{7} 、 \mathrm{Yb}_{2} \mathrm{Si}_{2} \mathrm{O}_{7}$ 和 $\mathrm{Lu}_{2} \mathrm{Si}_{2} \mathrm{O}_{7}$ 外, 其它 $\mathrm{RE}_{2} \mathrm{Si}_{2} \mathrm{O}_{7}$ 均具有至少两种多型结构, 且在高温下会 发生相转变。与 $\mathrm{RE}_{2} \mathrm{SiO}_{5}$ 相比, $\mathrm{RE}_{2} \mathrm{Si}_{2} \mathrm{O}_{7}$ 材料耐高温 腐蚀性能, 尤其是耐高温 CMAS 腐蚀性能相对不足。 $\mathrm{RE}_{2} \mathrm{Si}_{2} \mathrm{O}_{7}$ 优势在于热膨胀系数为 $(4 \sim 5) \times 10^{-6} \mathrm{~K}^{-1[35-36]}$, 与 $\mathrm{SiC}_{\mathrm{f}} / \mathrm{SiC}$ 复合材料基体较为匹配, 且 $\mathrm{RE}_{2} \mathrm{Si}_{2} \mathrm{O}_{7}$ 与 $\mathrm{Si}$ 或 $\mathrm{SiO}_{2}$ 化学相容性好, 高温条件下可以稳定共 存。总体来说, 目前已知的稀土硅酸盐材料各具性 能优势和不足, 作为 $\mathrm{EBC}$ 选材时仍然存在热应力失 配、高温相转变和耐高温 CMAS 腐蚀性能不足等问 题, 综合性能尚无法满足极端燃气环境中的长寿命 
服役要求。因此, $\mathrm{EBC}$ 用稀土硅酸盐材料的关键性 能优化和新体系探索仍是研究重点, 需要不断突破 创新。单稀土组元硅酸盐材料及其简单固溶体材料 的篎选和优化研究已经遇到瓶颈，能够实现的性能 调控空间较为有限, 难以满足未来环境障涂层的性 能要求。

高熵陶瓷和高熵化设计的新概念为人们在全系 稀土范围内设计多组元/高熵稀土硅酸盐材料, 开展 高熵稀土硅酸盐涂层材料的成分设计和性能调控提 供了新思路和新契机。已有研究表明, 稀土硅酸盐 材料抵抗燃气环境腐蚀、热膨胀系数和高温相稳定 等关键性能均与稀土元素种类直接相关，前半周期 和后半周期的轻或重稀土元素都能够对材料的宏观 性能起决定性作用且各具优点 ${ }^{[35-37]}$, 这为通过多稀 土组元/高熵化合物设计提供了可能性和指导性原 则。近年来, 有研究工作者尝试利用高熵策略来优 化稀土硅酸盐材料的性能。高熵稀土双硅酸盐 $\left(\mathrm{Yb}_{0.2} \mathrm{Y}_{0.2} \mathrm{Lu}_{0.2} \mathrm{Sc}_{0.2} \mathrm{Gd}_{0.2}\right)_{2} \mathrm{Si}_{2} \mathrm{O}_{7}$ 涂层在室温到 $1300{ }^{\circ} \mathrm{C}$ 范围内具有优异的相稳定性，与碳化硅基复合材料 的热膨胀匹配较好，并在 $1250{ }^{\circ} \mathrm{C}, 50 \% \mathrm{H}_{2} \mathrm{O}-50 \% \mathrm{O}_{2}$ 水 蒸气环境中表现出较优的抗水蒸气腐蚀性能 ${ }^{[38]}$ 。高 熵稀土单相硅酸盐 $\left(\mathrm{Yb}_{0.25} \mathrm{Y}_{0.25} \mathrm{Lu}_{0.25} \mathrm{Er}_{0.25}\right)_{2} \mathrm{SiO}_{5}$ 在室 温到 $1200{ }^{\circ} \mathrm{C}$ 范围内相稳定性好且显示出较强的热 膨胀各向异性，有可能通过控制涂层的择优取向实 现与碳化硅基复合材料基体的热膨胀匹配 ${ }^{[39]}$ 。总体 来说, 通过合理的高熵化设计, 有望实现稀土硅酸 盐的性能优化, 且性能调控空间较为广阔。本文主 要结合环境障涂层用稀土硅酸盐的关键性能局限, 重点介绍目前利用高熵化合物设计策略来实现稀土 硅酸盐材料热学、抗 CMAS 腐蚀和相稳定性等关键 性能提升的研究进展。

\section{2 稀土硅酸盐的高熵化设计与关键性 能调控典型案例}

\section{1 稀土硅酸盐的热学性能调控}

典型稀土单硅酸盐 $\mathrm{RE}_{2} \mathrm{SiO}_{5}$ 具有热导率低、相 稳定性好、耐水蒸气和 CMAS 腐蚀性能优等优点, 是极具性能优势的环境障涂层候选材料。但是稀土 单硅酸盐的热膨胀系数一般为 $(6.94 \sim 8.84) \times 10^{-6} \mathrm{~K}^{-1}$, 明显高于 $\mathrm{SiC}_{\mathrm{f}} / \mathrm{SiC}$ 复合材料 $\left((3.5 \sim 5.5) \times 10^{-6} \mathrm{~K}^{-1}\right)^{[34-35]}$ 。 为了将稀土单硅酸盐实际应用于环境障涂层, 必须 降低其热膨胀系数以实现良好的热匹配性。

Ren 等 ${ }^{[40]}$ 采用高熵化设计方法, 优选了抗水蒸
气腐蚀性能优异的 Ho、Er 元素，以及耐 CMAS 腐 蚀性能好的 $\mathrm{Y} 、 \mathrm{Yb}$ 元素, 制备了单相高摘稀土单硅 酸盐 $\left(\mathrm{Y}_{1 / 4} \mathrm{Ho}_{1 / 4} \mathrm{Er}_{1 / 4} \mathrm{Yb}_{1 / 4}\right)_{2} \mathrm{SiO}_{5}$ (简写为 $\left(4 \mathrm{RE}_{1 / 4}\right)_{2} \mathrm{SiO}_{5}$ ) 材料。从图 1 可见该材料为 $\mathrm{X} 2$ 结构 $\mathrm{RE}_{2} \mathrm{SiO}_{5}$, 且四 种稀土元素离子在原子尺度均匀随机分布，证明该 材料为单相高熵稀土单硅酸盐。且高熵 $\left(4 \mathrm{RE}_{1 / 4}\right)_{2} \mathrm{SiO}_{5}$ 在室温至 $1200{ }^{\circ} \mathrm{C}$ 范围的热膨胀系数明显低于四种 单组元稀土硅酸盐 $\mathrm{RE}_{2} \mathrm{SiO}_{5}(\mathrm{RE}=\mathrm{Y} 、 \mathrm{Ho} 、 \mathrm{Er}$ 和 $\mathrm{Yb})$, 降低幅度约 11\% 19\%(如图 2 所示)。此外，该 $\left(4 \mathrm{RE}_{1 / 4}\right)_{2} \mathrm{SiO}_{5}$ 的室温热导率较四种单组元稀土硅酸 盐 $\mathrm{RE}_{2} \mathrm{SiO}_{5}$ 降低了 $5.40 \%$ \%6.8\%。热膨胀系数和热 导率的协同降低，可使高熵 $\left(4 \mathrm{RE}_{1 / 4}\right)_{2} \mathrm{SiO}_{5}$ 的热膨胀 系数与 $\mathrm{SiC}_{\mathrm{f}} / \mathrm{SiC}$ 基体匹配良好, 在有效降低 $\mathrm{EBC}$ 应 力的同时，可以提供更好的热障防护作用。
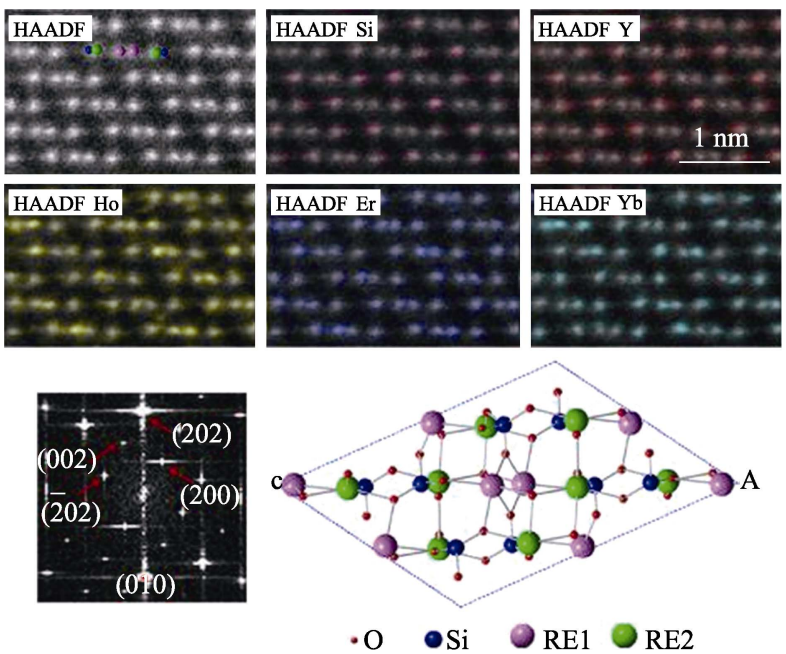

图 1 高熵稀土单硅酸盐 $\left(\mathrm{Y}_{1 / 4} \mathrm{Ho}_{1 / 4} \mathrm{Er}_{1 / 4} \mathrm{Yb}_{1 / 4}\right)_{2} \mathrm{SiO}_{5}$ 的 HAADFSTEM 照片及其原子尺度元素分布图 ${ }^{[40]}$

Fig. 1 HAADF-STEM image of high entropy $\left(\mathrm{Y}_{1 / 4} \mathrm{Ho}_{1 / 4} \mathrm{Er}_{1 / 4} \mathrm{Yb}_{1 / 4}\right)_{2}-$ $\mathrm{SiO}_{5}$, and EDS mapping of uniform spatial distributions for each element ${ }^{[40]}$

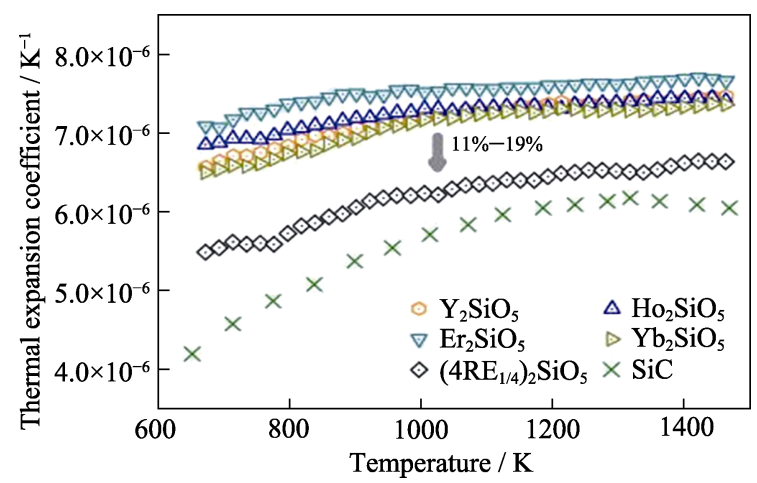

图 2 高熵稀土单硅酸盐 $\left(\mathrm{Y}_{1 / 4} \mathrm{Ho}_{1 / 4} \mathrm{Er}_{1 / 4} \mathrm{Yb}_{1 / 4}\right)_{2} \mathrm{SiO}_{5}$ 的热膨胀 系数随温度的变化 ${ }^{[40]}$

Fig. 2 Temperature dependent thermal expansion coefficient of high entropy $\left(\mathrm{Y}_{1 / 4} \mathrm{Ho}_{1 / 4} \mathrm{Er}_{1 / 4} \mathrm{Yb}_{1 / 4}\right)_{2} \mathrm{SiO}_{5}{ }^{[40]}$ 
Ridley 等 ${ }^{[41]}$ 研究了高熵化设计对于稀土单硅酸 盐热膨胀系数各向异性及热导率的影响。通过高温 $\mathrm{X}$ 射线衍射技术，对比研究了 $\mathrm{Sc}_{2} \mathrm{SiO}_{5} 、 \mathrm{Y}_{2} \mathrm{SiO}_{5}$ 、 $\mathrm{Nd}_{2} \mathrm{SiO}_{5} 、 \mathrm{Dy}_{2} \mathrm{SiO}_{5} 、 \mathrm{Er}_{2} \mathrm{SiO}_{5} 、 \mathrm{Yb}_{2} \mathrm{SiO}_{5}$ 和高熵 $(\mathrm{Sc}, \mathrm{Y}$, $\mathrm{Dy}, \mathrm{Er}, \mathrm{Yb})_{2} \mathrm{SiO}_{5}$ 高温热膨胀系数的各向异性差异。 研究结果发现稀土单硅酸盐材料的热膨胀系数各向 异性差异明显, 对单稀土组元(Sc、Y、Dy、Er 和 $\mathrm{Yb}) \mathrm{X} 2$ 相单硅酸盐来说, 从室温至 $1200{ }^{\circ} \mathrm{C}$, 其 $a$ 轴 热膨胀系数均低于 $3.64 \times 10^{-6} \mathrm{~K}^{-1}$, 而 $c$ 轴方向对应 的热膨胀系数通常为 $(9 \sim 10) \times 10^{-6} \mathrm{~K}^{-1}$ 。进一步通过放 电等离子烧结方法获得了高熵 $(\mathrm{Sc}, \mathrm{Y}, \mathrm{Dy}, \mathrm{Er}, \mathrm{Yb})_{2} \mathrm{SiO}_{5}$, 其各轴向之间的热膨胀系数依然表现出明显的各向 异性, 特别是 $a$ 轴和 $b$ 轴方向的膨胀系数以及材料 的平均线膨胀系数, 基本与各单组元硅酸盐材料对 应热膨胀系数的混合平均值保持一致。Ridley 等进 一步利用瞬态板热源法(Hot-disk technique)研究了 上述材料的导热性能。研究结果显示: 制备的高熵 $(\mathrm{Sc}, \mathrm{Y}, \mathrm{Dy}, \mathrm{Er}, \mathrm{Yb})_{2} \mathrm{SiO}_{5}$ 的室温热导率为 $1.06 \mathrm{~W} \cdot \mathrm{m}^{-1} \cdot \mathrm{K}^{-1}$, 低于对应单组元材料的热导率混合平均值, 且低于 所有对应单稀土组元硅酸盐材料。高熵 $(\mathrm{Sc}, \mathrm{Y}, \mathrm{Dy}$, $\mathrm{Er}, \mathrm{Yb})_{2} \mathrm{SiO}_{5}$ 的热导率进一步降低, 有助于提升其应 用于环境障涂层时的热障功能, 为稀土硅酸盐作为 多功能热障/环障涂层材料的创新应用提供支撑。

Chen 等 ${ }^{[21]}$ 也重点关注了高熵化对于稀土单硅酸 盐热膨胀各向异性的影响。通过固态反应烧结方法成 功制备了单相纯净的高熵 $\left(\mathrm{Yb}_{0.25} \mathrm{Y}_{0.25} \mathrm{Lu}_{0.25} \mathrm{Er}_{0.25}\right)_{2} \mathrm{SiO}_{5}$ 稀土单硅酸盐材料, 利用高温 $\mathrm{X}$ 射线衍射及晶体结 构精修方法研究了该材料从室温至 $1473 \mathrm{~K}$ 的热膨 胀行为各向异性。研究结果显示, 与单组元稀土硅 酸盐材料类似, 该高熵稀土硅酸盐材料的热膨胀系 数也展现出明显的各向异性: 其 $a$ 轴热膨胀系数为 $2.57 \times 10^{-6} \mathrm{~K}^{-1}, b$ 轴方向热膨胀系数为 $8.07 \times 10^{-6} \mathrm{~K}^{-1}$, $c$ 轴方向为 $9.98 \times 10^{-6} \mathrm{~K}^{-1}$, 其平均线膨胀系数为 $7.63 \times 10^{-6} \mathrm{~K}^{-1}$ 。基于上述研究, Chen 等进一步提出利 用稀土单硅酸盐热膨胀行为各向异性差异, 通过有 目的性地控制稀土单硅酸盐涂层的晶体学取向, 可 以实现涂层与金属/陶瓷基体热膨胀系数匹配的设想。

此外, Turcer 等 $^{[42]}$ 利用高熵化方法对稀土双硅 酸盐的热导率进行了调控研究。通过选取 $Y 、 Y b$ 、 Sc、 $\mathrm{Gd}$ 和 $\mathrm{Lu}$ 五种稀土元素, 利用放电等离子烧结 技术制备了高熵 $\left(\mathrm{Y}_{0.2} \mathrm{Yb}_{0.2} \mathrm{Sc}_{0.2} \mathrm{Gd}_{0.2} \mathrm{Lu}_{0.2}\right)_{2} \mathrm{Si}_{2} \mathrm{O}_{7}$ 稀土 双硅酸盐材料, 并利用闪光法研究了其室温至 $1000{ }^{\circ} \mathrm{C}$ 的热导率。研究结果显示, 该高熵材料具有 单相 $\beta$ 型结构, 其热导率显著低于对应的单组元稀 土双硅酸盐及简单固溶体材料, 如: $\beta-\mathrm{Yb}_{2} \mathrm{Si}_{2} \mathrm{O}_{7}$ 、 $\beta-\left(\mathrm{Y}_{0.1} \mathrm{Yb}_{0.9}\right)_{2} \mathrm{Si}_{2} \mathrm{O}_{7}$ 和 $\beta-\left(\mathrm{Y}_{0.5} \mathrm{Yb}_{0.5}\right)_{2} \mathrm{Si}_{2} \mathrm{O}$ 。特别是
$1000{ }^{\circ} \mathrm{C}$ 时 $\beta$ - $\left(\mathrm{Y}_{0.2} \mathrm{Yb}_{0.2} \mathrm{Sc}_{0.2} \mathrm{Gd}_{0.2} \mathrm{Lu}_{0.2}\right)_{2} \mathrm{Si}_{2} \mathrm{O}_{7}$ 的热导率 仅为 $1.52 \mathrm{~W} \cdot \mathrm{m}^{-1} \cdot \mathrm{K}^{-1}$, 有望为环境障涂层提供优异 的隔热性能。

\section{2 稀土硅酸盐耐 CMAS 腐蚀性能调控}

稀土双硅酸盐具有与 $\mathrm{SiC}_{\mathrm{f}} / \mathrm{SiC}$ 陶瓷基复合材料 相匹配的热膨胀系数、与 $\mathrm{Si} / \mathrm{SiO}_{2}$ 良好的化学相容性 以及优异的损伤容限, 是极具潜力的环境障涂层候 选材料。受限于稀土双硅酸盐的多型相转变, 目前 只有 $\beta-\mathrm{Yb}_{2} \mathrm{Si}_{2} \mathrm{O}_{7} 、 \beta-\mathrm{Lu}_{2} \mathrm{Si}_{2} \mathrm{O}_{7}$ 和 $\beta-\mathrm{Sc}_{2} \mathrm{Si}_{2} \mathrm{O}_{7}$ 等少数材 料被优先考虑用于环境障涂层应用。Poerschke 等 ${ }^{[43]}$ 对比了几种环境障涂层材料在 $1300{ }^{\circ} \mathrm{C}$ 与 $\mathrm{CMAS}$ 反 应的侵入深度与时间的关系, 发现稀土硅酸盐抗 CMAS 腐蚀能力优于第二代环障涂层材料 BSAS, 且稀土双硅酸盐抗 CMAS 腐蚀能力略低于对应的 稀土单硅酸盐材料。 $\mathrm{Liu}$ 等 ${ }^{[44]}$ 研究了几种 $\mathrm{RE}_{2} \mathrm{Si}_{2} \mathrm{O}_{7}$ 在 $1400{ }^{\circ} \mathrm{C}$ 水蒸气环境中与 CMAS 的腐蚀反应, 发 现 $\mathrm{RE}_{2} \mathrm{Si}_{2} \mathrm{O}_{7}$ 与 $\mathrm{CMAS}$ 发生较为剧烈的反应, 生成磷 灰石相, 且反应层中存在大量气孔。Tian 等 ${ }^{[45]}$ 研究 了 $\gamma-\mathrm{Y}_{2} \mathrm{Si}_{2} \mathrm{O}_{7} 、 \beta-\mathrm{Yb}_{2} \mathrm{Si}_{2} \mathrm{O}_{7}$ 和 $\beta-\mathrm{Lu}_{2} \mathrm{Si}_{2} \mathrm{O}_{7}$ 在 1300 和 $1500{ }^{\circ} \mathrm{C}$ 下耐 CMAS 腐蚀 $50 \mathrm{~h}$ 的性能, 发现 $1300{ }^{\circ} \mathrm{C}$ 下 $\beta-\mathrm{Yb}_{2} \mathrm{Si}_{2} \mathrm{O}_{7}$ 和 $\beta-\mathrm{Lu}_{2} \mathrm{Si}_{2} \mathrm{O}_{7}$ 样品均与 CMAS 发生剧 烈反应, 其中 $\gamma-\mathrm{Y}_{2} \mathrm{Si}_{2} \mathrm{O}_{7}$ 在 $1300{ }^{\circ} \mathrm{C}$ 下抗 CMAS 腐蚀 能力较好, 但其在高温长时间腐蚀环境中会发生相 转变, 不适合应用于高稳定性环境障涂层。Tian 等 ${ }^{[45]}$ 的实验结果还显示 $\gamma-\mathrm{Y}_{2} \mathrm{Si}_{2} \mathrm{O}_{7} 、 \beta-\mathrm{Yb}_{2} \mathrm{Si}_{2} \mathrm{O}_{7}$ 和 $\beta-\mathrm{Lu}_{2} \mathrm{Si}_{2} \mathrm{O}_{7}$ 均无法有效抵御 $1500{ }^{\circ} \mathrm{C}$ 下 $\mathrm{CMAS}$ 长时间 腐蚀, 熔融 CMAS 沿晶界向样品内部剧烈渗透, 且 反应后样品截面发现大量的气泡和横向粗裂纹。Turcer 等 ${ }^{[46-47]}$ 研究了 $1500{ }^{\circ} \mathrm{C}$ 下 $\gamma-\mathrm{Y}_{2} \mathrm{Si}_{2} \mathrm{O}_{7} 、 \beta-\mathrm{Yb}_{2} \mathrm{Si}_{2} \mathrm{O}_{7}$ 和 $\beta-\mathrm{Sc}_{2} \mathrm{Si}_{2} \mathrm{O}_{7}$ 的耐 CMAS 腐蚀性能, 结果与 Tian 等的 结论类似, 在 $1500{ }^{\circ} \mathrm{C}$ 下 CMAS 腐蚀 $24 \mathrm{~h}$ 后材料均 产生了明显失效。

为提高稀土双硅酸盐的耐 CMAS 腐蚀性能, Sun 等 ${ }^{[48]}$ 选取了 $\mathrm{Er} 、 \mathrm{Tm} 、 \mathrm{Yb}$ 和 $\mathrm{Lu}$ 四种元素, 制备了高熵 $\left(\mathrm{Er}_{1 / 4} \mathrm{Tm}_{1 / 4} \mathrm{Yb}_{1 / 4} \mathrm{Lu}_{1 / 4}\right)_{2} \mathrm{Si}_{2} \mathrm{O}_{7}$ (后续简写为 $\left(4 \mathrm{RE}_{1 / 4}\right)_{2} \mathrm{Si}_{2} \mathrm{O}_{7}$ ) 稀土双硅酸盐, 并开展了腐蚀性能研究。他们通过 无压合成一热压烧结两步法获得了单相致密的高熵 (4RE $\left.\mathrm{R}_{1 / 4}\right)_{2} \mathrm{Si}_{2} \mathrm{O}_{7}$ 材料, 并研究了其 $1500{ }^{\circ} \mathrm{C}$ 的耐 CMAS(摩尔比为 $33 \mathrm{CaO}-9 \mathrm{MgO}-13 \mathrm{AlO}_{1.5}-45 \mathrm{SiO}_{2}$ ) 腐 蚀性能。研究结果如图 3 所示, 经过 $1500{ }^{\circ} \mathrm{C}$ 高温 CMAS 腐蚀反应 4 和 $50 \mathrm{~h}$ 后, 样品表面均主要分布 着板条状反应产物, 结合 $\mathrm{X}$ 射线衍射和能谱分析结 果判断该产物为磷灰石结构的 $\mathrm{Ca}_{2} \mathrm{RE}_{8}\left(\mathrm{SiO}_{4}\right)_{6} \mathrm{O}_{2}$ 相。 放大图 3(b,d)显示, 经高温 CMAS 腐蚀 4 和 $50 \mathrm{~h}$ 后, 样品表面依然有黑色祄度的 CMAS 残留，且二者形 
貌有明显区别：(1)腐蚀 $4 \mathrm{~h}$ 后的样品表面残余 CMAS 明显多于腐蚀 $50 \mathrm{~h}$ 样品; (2)腐蚀反应 $4 \mathrm{~h}$ 后生 成的磷灰石相多呈现中空的细长管状形貌，长管中 心依然残留有未消耗的 CMAS; 腐蚀反应 $50 \mathrm{~h}$ 后析 出的磷灰石相形貌上更为粗大, 且中心有 CMAS 残留 的板条结构明显减少。图 4 为高熵 $\left(4 \mathrm{RE}_{1 / 4}\right)_{2} \mathrm{Si}_{2} \mathrm{O}_{7}$ 经 $1500{ }^{\circ} \mathrm{C}$ 下 $\mathrm{CMAS}$ 腐蚀后样品截面扫描照片。样品 经 CMAS 腐蚀反应 4 和 $50 \mathrm{~h}$ 后，稀土硅酸盐基体(图 4(d) S2)与 CMAS 的反应区之间明显存在平直、完 整的反应界面，且两个样品的反应区厚度相差不大， 均约为 $300 \mu \mathrm{m}$ 。与腐蚀后表面观察结果类似, 截面 样品的反应区内同样可观察到板条状的磷灰石相(图 4(d) S3)和残余 CMAS(图 4(d) S1)。值得注意的是, $\left(4 \mathrm{RE}_{1 / 4}\right)_{2} \mathrm{Si}_{2} \mathrm{O}_{7}$ 与 $\mathrm{CMAS}$ 在 $1500{ }^{\circ} \mathrm{C}$ 反应 $50 \mathrm{~h}$ 后, 并 没有观察到 Tian 和 Turcer 等发现的 CMAS 沿晶界
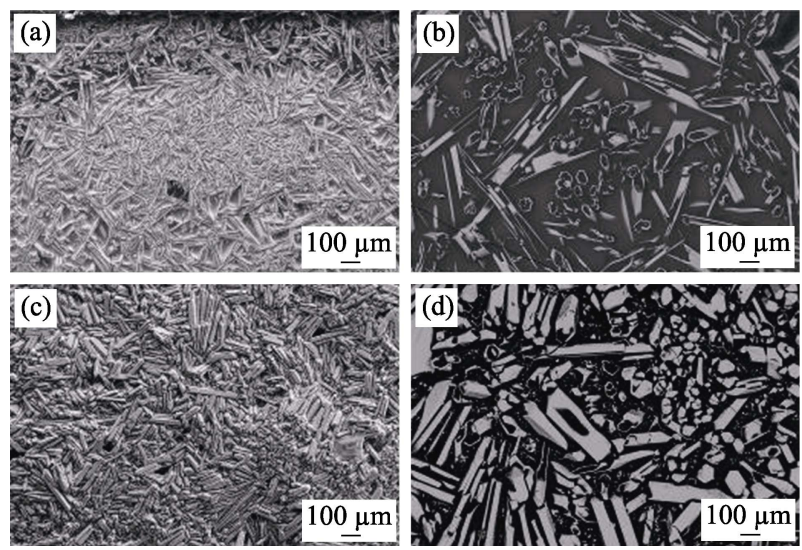

图 3 高熵稀土双硅酸盐 $\left(\mathrm{Er}_{1 / 4} \mathrm{Tm}_{1 / 4} \mathrm{Yb}_{1 / 4} \mathrm{Lu}_{1 / 4}\right)_{2} \mathrm{Si}_{2} \mathrm{O}_{7}$ 在 $1500{ }^{\circ} \mathrm{C}$ 高 温 CMAS 腐蚀 $(\mathrm{a} \sim \mathrm{b}) 4 \mathrm{~h}$ 和 $(\mathrm{c} \sim \mathrm{d}) 50 \mathrm{~h}$ 后样品表面形貌 ${ }^{[48]}$

Fig. 3 Surface observations of high entropy $\left(\mathrm{Er}_{1 / 4} \mathrm{Tm}_{1 / 4} \mathrm{Yb}_{1 / 4} \mathrm{Lu}_{1 / 4}\right)_{2} \mathrm{Si}_{2} \mathrm{O}_{7}$ after CMAS corrosion at $1500{ }^{\circ} \mathrm{C}$ for $4 \mathrm{~h}(\mathrm{a}-\mathrm{b})$ and $50 \mathrm{~h}(\mathrm{c}-\mathrm{d})^{[48]}$
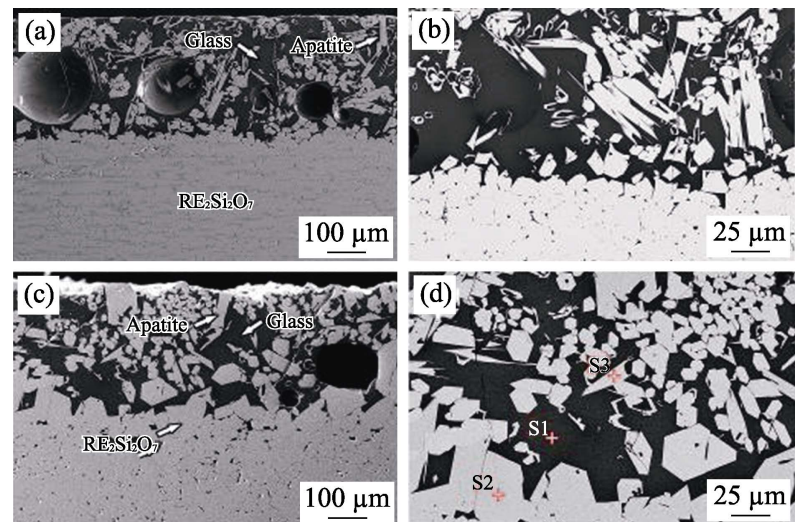

图4 高熵稀土双硅酸盐 $\left(\mathrm{Er}_{1 / 4} \mathrm{Tm}_{1 / 4} \mathrm{Yb}_{1 / 4} \mathrm{Lu}_{1 / 4}\right)_{2} \mathrm{Si}_{2} \mathrm{O}_{7}$ 在 $1500{ }^{\circ} \mathrm{C}$ 下 CMAS 腐蚀 $(\mathrm{a} \sim \mathrm{b}) 4 \mathrm{~h}$ 和 $(\mathrm{c} \sim \mathrm{d}) 50 \mathrm{~h}$ 截面形貌 ${ }^{[48]}$

Fig. 4 Observations of the reaction front in the cross-sections of high entropy $\left(\mathrm{Er}_{1 / 4} \mathrm{Tm}_{1 / 4} \mathrm{Yb}_{1 / 4} \mathrm{Lu}_{1 / 4}\right)_{2} \mathrm{Si}_{2} \mathrm{O}_{7}$ after CMAS corrosion at $1500{ }^{\circ} \mathrm{C}$ for $4 \mathrm{~h}(\mathrm{a}, \mathrm{b})$ and $50 \mathrm{~h}(\mathrm{c}, \mathrm{d})^{[48]}$
完全渗入基体的现象，也没有在稀土双硅酸盐基体 中观察到明显的横向裂纹。实验结果表明 $1500{ }^{\circ} \mathrm{C}$ 下 该高熵稀土双硅酸盐与 CMAS 的腐蚀反应较为缓慢, 可实现多稀土元素抵抗 CMAS 腐蚀的协同优化效应。

\section{3 稀土硅酸盐结构稳定相调控}

已知稀土双硅酸盐存在多达 7 种多型结构,除 $\beta-\mathrm{Sc}_{2} \mathrm{Si}_{2} \mathrm{O}_{7} 、 \beta-\mathrm{Yb}_{2} \mathrm{Si}_{2} \mathrm{O}_{7}$ 和 $\beta-\mathrm{Lu}_{2} \mathrm{Si}_{2} \mathrm{O}_{7}$ 外, 其它稀土 双硅酸盐均存在高温多晶型相变。考虑到 $\mathrm{Sc}$ 和 $\mathrm{Lu}$ 元素价格昂贵, 目前 $\mathrm{EBC}$ 用稀土双硅酸盐研究多集 中于 $\beta-\mathrm{Yb}_{2} \mathrm{Si}_{2} \mathrm{O}_{7}$ 。因此, 抑制高温多晶型相变是稀 土双硅酸盐应用于 $\mathrm{EBC}$ 的关键性能突破方向。已有 研究表明, 稀土双硅酸盐的晶型与稀土离子半径大 小存在明显依赖关系 ${ }^{[49]}$, 考虑到多稀土组元固溶体 离子半径的调制作用以及高熵化合物的熵稳定作用, 高熵化可能是提高稀土双硅酸盐相稳定性的有效途 径。结合前述高熵化设计对于稀土双硅酸盐耐 CMAS 腐蚀性能提升的探索结果，合理的高熵化策 略有望为 $\mathrm{EBC}$ 用稀土双硅酸盐材料的成分篮选和 性能调控提供更广阔的设计空间，推动其在下一代 环境障涂层的创新应用。

Dong 等 ${ }^{[38]}$ 研究了 $\beta$ 结构高熵稀土双硅酸盐的 相稳定性，他们首先利用溶胶凝胶法获得了 $\beta$ 结构 $\left(\mathrm{Y}_{0.2} \mathrm{Yb}_{0.2} \mathrm{Sc}_{0.2} \mathrm{Gd}_{0.2} \mathrm{Lu}_{0.2}\right)_{2} \mathrm{Si}_{2} \mathrm{O}_{7}$ 高熵稀土双硅酸盐粉 体材料, 利用热重一差热分析研究了该材料的高温 稳定性。研究结果显示, 在室温至 $1300{ }^{\circ} \mathrm{C}$ 范围内, 没有发现明显的吸热/放热峰, 该材料展现出优异的 高温相稳定性。Dong 等进一步研究了该粉体材料在 $1400{ }^{\circ} \mathrm{C} 、 50 \% \mathrm{H}_{2} \mathrm{O}-50 \% \mathrm{O}_{2}$ 水蒸气腐蚀条件下的稳定 性及耐腐蚀性能, 并将该材料涂覆在 $\mathrm{C}_{\mathrm{f}} / \mathrm{SiC}$ 基体上, 研究了该高熵稀土硅酸盐作为环境障涂层材料在 $1250{ }^{\circ} \mathrm{C}$ 下耐 $50 \% \mathrm{H}_{2} \mathrm{O}-50 \% \mathrm{O}_{2}$ 水蒸气腐蚀性能。研究 结果表明，该高熵稀土硅酸盐粉体经 $1400{ }^{\circ} \mathrm{C}$ 、 $50 \% \mathrm{H}_{2} \mathrm{O}-50 \% \mathrm{O}_{2}$ 水蒸气腐蚀 $200 \mathrm{~h}$ 后，依然保持 $\beta$ 结构, 展现出优异的相稳定性。同时, 该高摘稀土双 硅酸盐作为 $\mathrm{C}_{\mathrm{f}} / \mathrm{SiC}$ 材料的环境障涂层, 经 $1250{ }^{\circ} \mathrm{C}$ 、 $50 \% \mathrm{H}_{2} \mathrm{O}-50 \% \mathrm{O}_{2}$ 腐蚀 $300 \mathrm{~h}$ 后, 依然展现出优异的 相稳定性和耐水蒸气腐蚀性能。

Sun 等 ${ }^{[50]}$ 利用高熵化策略对 $\gamma$ 结构稀土双硅酸 盐进行了研究，通过选定 $G d 、 T b 、 D y 、 T m 、 Y b$ 和 $\mathrm{Lu}$ 六种稀土元素，利用无压合成法获得了 $\left(\mathrm{Gd}_{1 / 6} \mathrm{~Tb}_{1 / 6} \mathrm{Dy}_{1 / 6} \mathrm{Tm}_{1 / 6} \mathrm{Yb}_{1 / 6} \mathrm{Lu}_{1 / 6}\right)_{2} \mathrm{Si}_{2} \mathrm{O}_{7}$ (后续简写为 $\left.\left(6 \mathrm{RE}_{1 / 6}\right)_{2} \mathrm{Si}_{2} \mathrm{O}_{7}\right)$ 粉体材料。图 5(a) 所示为制备的高熵 稀土双硅酸盐及对应单稀土组元双硅酸盐的 XRD 

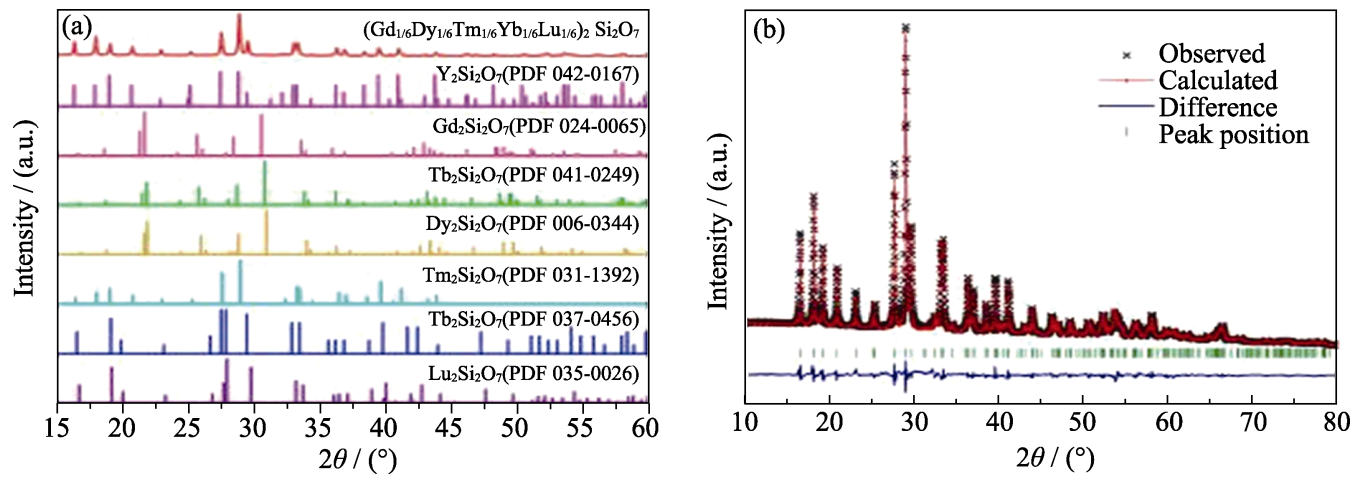

图 5 (a)高熵稀土双硅酸盐 $\left(\mathrm{Gd}_{1 / 6} \mathrm{~Tb}_{1 / 6} \mathrm{Dy}_{1 / 6} \mathrm{Tm}_{1 / 6} \mathrm{Yb}_{1 / 4} \mathrm{Lu}_{1 / 6}\right)_{2} \mathrm{Si}_{2} \mathrm{O}_{7}$ 及 $\mathrm{RE}_{2} \mathrm{Si}_{2} \mathrm{O}_{7}(\mathrm{RE}=\mathrm{Y}, \mathrm{Gd}, \mathrm{Tb}, \mathrm{Dy}, \mathrm{Tm}, \mathrm{Yb}$ 和 $\mathrm{Lu})$ 的 $\mathrm{XRD}$ 图谱和(b)高熵稀土双硅酸盐 $\left(\mathrm{Gd}_{1 / 6} \mathrm{~Tb}_{1 / 6} \mathrm{Dy}_{1 / 6} \mathrm{Tm}_{1 / 6} \mathrm{Yb}_{1 / 4} \mathrm{Lu}_{1 / 6}\right)_{2} \mathrm{Si}_{2} \mathrm{O}_{7}$ 的 XRD 图谱 Rietveld 精修结果 ${ }^{[50]}$

Fig. 5 (a) XRD patterns of $\left(\mathrm{Gd}_{1 / 6} \mathrm{~Tb}_{1 / 6} \mathrm{Dy}_{1 / 6} \mathrm{Tm}_{1 / 6} \mathrm{Yb}_{1 / 4} \mathrm{Lu}_{1 / 6}\right)_{2} \mathrm{Si}_{2} \mathrm{O}_{7}$, along with the standard XRD patterns of $\mathrm{RE}_{2} \mathrm{Si}_{2} \mathrm{O}_{7}(\mathrm{RE}=\mathrm{Y}$, $\mathrm{Gd}, \mathrm{Tb}$, Dy, Tm, Yb and Lu) and (b) Rietveld refinement of XRD pattern for $\left(\mathrm{Gd}_{1 / 6} \mathrm{~Tb}_{1 / 6} \mathrm{Dy}_{1 / 6} \mathrm{Tm}_{1 / 6} \mathrm{Yb}_{1 / 4} \mathrm{Lu}_{1 / 6}\right)_{2} \mathrm{Si}_{2} \mathrm{O}_{7}[50]$

数据, 可见该材料为单相 $\gamma$ 晶型稀土双硅酸盐。特别 需要指出的是: $\mathrm{Gd}$ 、 $\mathrm{Tb}$ 和 $\mathrm{Dy}$ 三种稀土元素对应的 高温稳定稀土双硅酸盐为 $\delta$ 相结构, 而 $\mathrm{Tm} 、 \mathrm{Yb}$ 和 $\mathrm{Lu}$ 三种元素对应的高温稳定结构为 $\beta$ 相结构。通过 Rietveld 方法对 $\left(6 \mathrm{RE}_{1 / 6}\right)_{2} \mathrm{Si}_{2} \mathrm{O}_{7}$ 的 XRD 数据进行精修, 结果如图 5(b)所示, 确定该材料为新出现的 $\gamma$ 相结 构: 属于 $\mathrm{P} 2{ }_{1} / \mathrm{c}$ 空间群, 晶胞参数为 $a=0.4683 \mathrm{~nm}$; $b=1.083 \mathrm{~nm} ; c=0.5572 \mathrm{~nm} ; \beta=95.98^{\circ}$ 。

Sun 等 ${ }^{[50]}$ 进一步利用热压烧结方法成功制备了 $\left(6 \mathrm{RE}_{1 / 6}\right)_{2} \mathrm{Si}_{2} \mathrm{O}_{7}$ 块体样品, 从图 6(a) 可见, 所得样品 为均匀致密的块体材料。进一步利用高角环形暗场 像一扫描透射电子成像技术(HAADF-STEM)对制备 样品进行原子尺度元素分析, 结果如图 6(b)所示, 六种稀土元素均匀分布在稀土元素的格点位置, 且 完全无序固溶，可以断定该 $\left(6 \mathrm{RE}_{1 / 6}\right)_{2} \mathrm{Si}_{2} \mathrm{O}_{7}$ 为一种高
熵陶瓷。该工作还展示了新奇的高熵相稳定结果： 与传统高熵合金晶体结构通常与某种或某几种单组 元合金结构保持一致的情况不同，该 $\gamma-\left(6 \mathrm{RE}_{1 / 6}\right)_{2} \mathrm{Si}_{2} \mathrm{O}_{7}$ 的晶体结构有别于对应单稀土组元硅酸盐的稳定结 构 ( $\beta$ 和 $\delta$ 相结构), 通过高熵化设计实现了一种“无中 生有”的全新相稳定模式。

Sun 等 ${ }^{[50]}$ 还采用热重 - 差热分析研究了 $\left(6 \mathrm{RE}_{1 / 6}\right)_{2} \mathrm{Si}_{2} \mathrm{O}_{7}$ 的高温稳定性, 研究结果如图 7(a)所 示：该材料从室温至 $1600{ }^{\circ} \mathrm{C}$ 表现出优异的热稳定 性和晶体结构稳定性。在 1800 和 $1900{ }^{\circ} \mathrm{C}$ 下对其热 处理 $2 \mathrm{~h}$, 然后利用 $\mathrm{X}$ 射线衍射对冷却后的样品进 行相分析, 如图 7(b)所示。热处理样品的 XRD 衍射 峰均对应于 $\gamma$ 型稀土双硅酸盐, 未检测到其它物相 或多型相的衍射峰，表明 $\left(6 \mathrm{RE}_{1 / 6}\right)_{2} \mathrm{Si}_{2} \mathrm{O}_{7}$ 在 1800 和 $1900{ }^{\circ} \mathrm{C}$ 高温下依然具有优异的晶体结构稳定性。 (a)

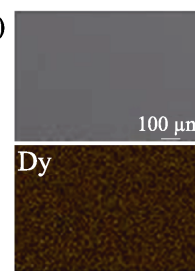

(b)

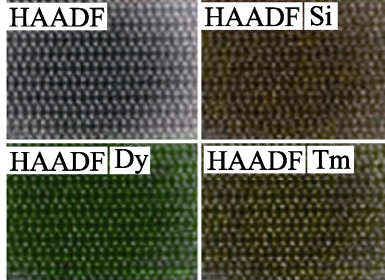

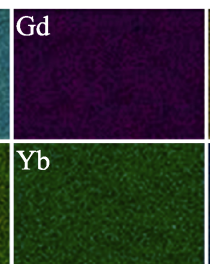

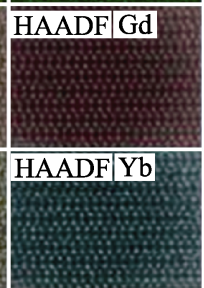

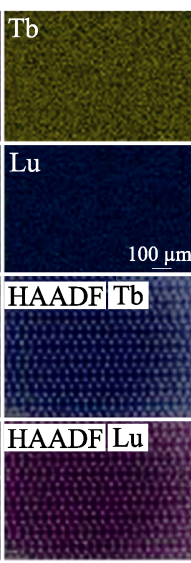

(c)

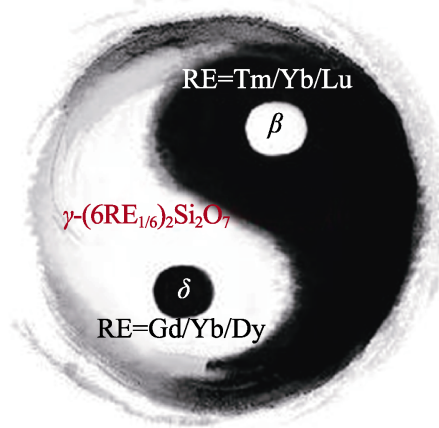

图 6 高熵稀土双硅酸盐 $\left(\mathrm{Gd}_{1 / 6} \mathrm{~Tb}_{1 / 6} \mathrm{Dy}_{1 / 6} \mathrm{Tm}_{1 / 6} \mathrm{Yb}_{1 / 4} \mathrm{Lu}_{1 / 6}\right)_{2} \mathrm{Si}_{2} \mathrm{O}_{7}$ 的(a) SEM 照片和各元素分布的面扫描能谱分析;

(b) HAADF-STEM 图像及原子尺度元素分布; (c)稀土双硅酸盐全新相稳定模式示意图 ${ }^{[50]}$

Fig. 6 (a) SEM image of $\left(\mathrm{Gd}_{1 / 6} \mathrm{~Tb}_{1 / 6} \mathrm{Dy}_{1 / 6} \mathrm{Tm}_{1 / 6} \mathrm{Yb}_{1 / 4} \mathrm{Lu}_{1 / 6}\right)_{2} \mathrm{Si}_{2} \mathrm{O}_{7}$ surface with EDS mappings of Si, Gd, Tb, Dy, Tm, Yb and Lu, (b) STEM high angle annular dark field (HAADF) image and corresponding selected compositional EDS maps of high entropy $\left(\mathrm{Gd}_{1 / 6} \mathrm{~Tb}_{1 / 6} \mathrm{Dy}_{1 / 6} \mathrm{Tm}_{1 / 6} \mathrm{Yb}_{1 / 4} \mathrm{Lu}_{1 / 6}\right)_{2} \mathrm{Si}_{2} \mathrm{O}_{7}$, and (c) schematic diagram of the phase formation of $\left(6 \mathrm{RE}_{1 / 6}\right)_{2} \mathrm{Si}_{2} \mathrm{O}_{7}{ }^{[50]}$ 

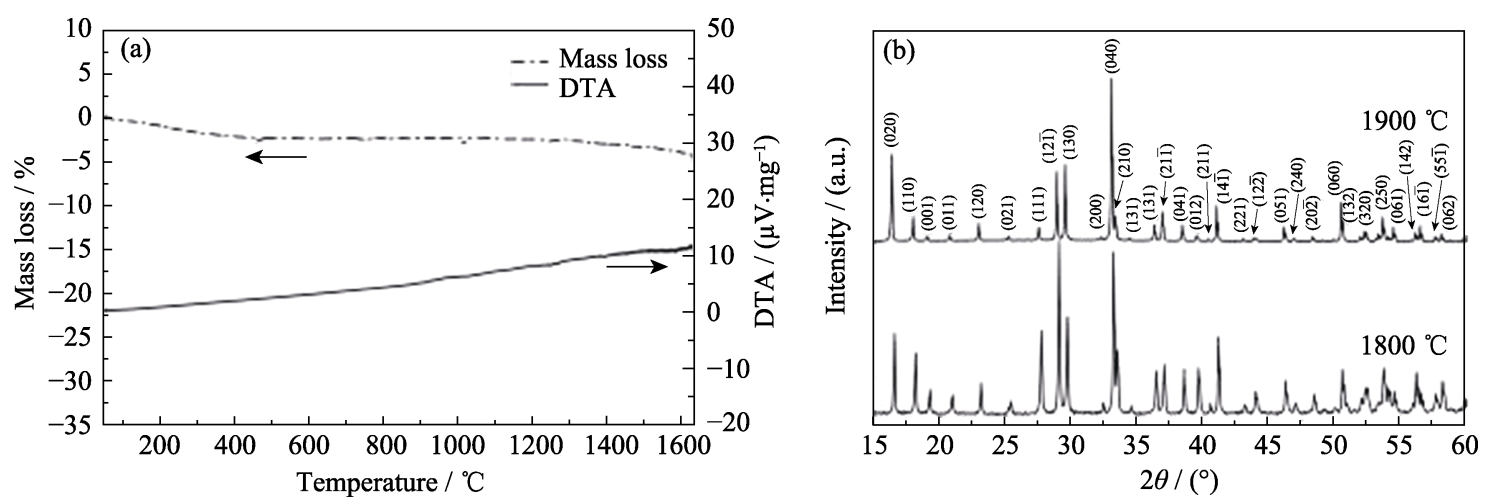

图 7 高熵稀土双硅酸盐 $\left(\mathrm{Gd}_{1 / 6} \mathrm{~Tb}_{1 / 6} \mathrm{Dy}_{1 / 6} \mathrm{Tm}_{1 / 6} \mathrm{Yb}_{1 / 4} \mathrm{Lu}_{1 / 6}\right)_{2} \mathrm{Si}_{2} \mathrm{O}_{7}$ 的(a)热重一差热分析曲线和 (b) 1800 及 $1900{ }^{\circ} \mathrm{C}$ 热处理 $2 \mathrm{~h}$ 后样品的 XRD 图谱 ${ }^{[50]}$

Fig. 7 (a) TG/DTA curves of $\left(\mathrm{Gd}_{1 / 6} \mathrm{~Tb}_{1 / 6} \mathrm{Dy}_{1 / 6} \mathrm{Tm}_{1 / 6} \mathrm{Yb}_{1 / 4} \mathrm{Lu}_{1 / 6}\right)_{2} \mathrm{Si}_{2} \mathrm{O}_{7}$ and (b) XRD patterns of specimens after being heat-treated at 1800 and $1900{ }^{\circ} \mathrm{C}$ for $2 \mathrm{~h}^{[50]}$

熔点测试结果显示, $\left(6 \mathrm{RE}_{1 / 6}\right)_{2} \mathrm{Si}_{2} \mathrm{O}_{7}$ 的熔点大约 为 $1907 \sim 1935{ }^{\circ} \mathrm{C}$ 。图 8 汇总了稀土双硅酸盐的多晶 型转变温度及熔点, 多型相结构由不同的颜色表示, 柱状图顶端对应温度表示材料的熔点 ${ }^{[47]}$ 。对比发现 $\left(6 \mathrm{RE}_{1 / 6}\right)_{2} \mathrm{Si}_{2} \mathrm{O}_{7}$ 不但在全温域范围内保持晶体结构稳 定性, 而且具有稀土双硅酸盐材料家族中最高的熔 点，展现出优异的晶体结构稳定性。

\section{3 结束语}

高熵化设计策略在稀土硅酸盐材料的热学性能 (低热膨胀系数和热导率)调控、耐高温低熔点氧化 物(CMAS) 腐蚀抗性提升以及高温相/结构稳定性优 化等方面的应用，成功避免或克服了现有稀土硅酸 盐材料作为环境障涂层应用的性能局限, 为 $\mathrm{EBC}$ 用 稀土硅酸盐材料的关键性能优化和创新设计提供了 新途径。但是后续研究仍需针对多稀土组元/高熵稀 土硅酸盐开展系统研究, 从稀土硅酸盐材料的结 构、成分与力学、热学和腐蚀性能的内禀关联和协

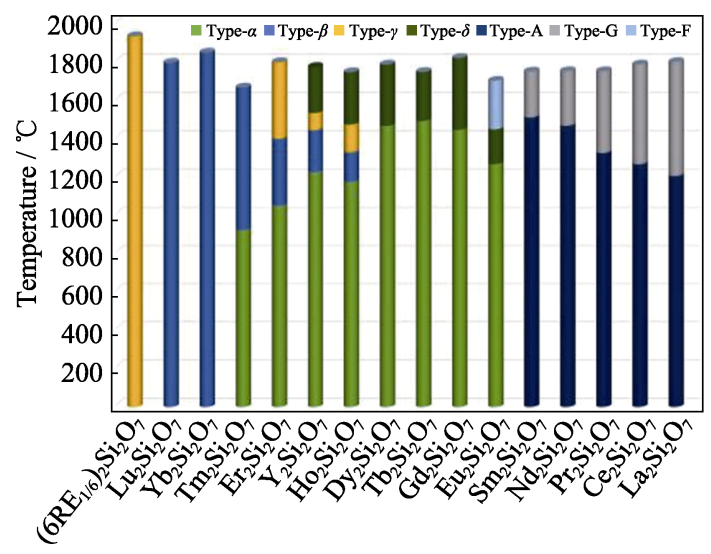

图 8 稀土双硅酸盐多晶型转变温度及熔点示意图 ${ }^{[50]}$

Fig. 8 Schematics of the polymorphic transformation temperatures and melting points of $\mathrm{RE}_{2} \mathrm{Si}_{2} \mathrm{O}_{7}$ disilicates ${ }^{[50]}$
同作用机制开展深入探索, 基于高熵化设计的科学 思路，探究高熵稀土硅酸盐的形成机理和固溶体稳 定控制机理，明晰稀土组元差异对于材料晶格畸变 和构型熵的影响机制，探索基于稀土组元内禀特性 的高熵稀土硅酸盐的性能调制原则，提出多功能热 障/环境障涂层用稀土硅酸盐材料综合性能调控设 计的新途径，实现航空发动机用陶瓷基复合材料热 结构部件的热障/环境障涂层的设计、选材与性能突 破, 为环境障涂层用稀土硅酸盐的发展提供新材料 储备和关键技术支撑。

\section{参考文献:}

[1] TSAI M H, YEH J W. High-entropy alloys: a critical review. Materials Research Letters, 2014, 2(3): 107-123.

[2] MIRACLE D B, SENKOV O N. A critical review of high entropy alloys and related concepts. Acta Materialia, 2017, 122: 448-511.

[3] YEH J W, CHEN S K, LIN S J, et al. Nanostructured high-entropy alloys with multiple principal elements: novel alloy design concepts and outcomes. Advanced Engineering Materials, 2004, 6(5): 299-303.

[4] HUANG P K, YEH J W, SHUN T T, et al. Multi-principal-element alloys with improved oxidation and wear resistance for thermal spray coating. Advanced Engineering Materials, 2004, 6(1/2): 74-78.

[5] TONG C J, CHEN Y L, CHEN S K, et al. Microstructure characterization of $\mathrm{Al}_{x} \mathrm{CoCrCuFeNi}$ high-entropy alloy system with multiprincipal elements. Metallurgical and Materials Transactions A, 2005, 36(4): 881-893.

[6] ZHANG Y, ZUO T T, TANG Z, et al. Microstructures and properties of high-entropy alloys. Progress in Materials Science, 2014, 61: 1-93.

[7] MURTY B S, YEH J W, RANGANATHAN S. High-entropy Alloys. London: Elsevier, 2014.

[8] ZHANG Y, ZUO T T, CHENG Y Q, et al. High-entropy alloys with high saturation magnetization, electrical resistivity, and malleability. Scientific Reports, 2013, 3: 1455.

[9] CHUANG M H, TSAI M H, WANG W R, et al. Microstructure and wear behavior of $\mathrm{Al}_{x} \mathrm{Co}_{1.5} \mathrm{CrFeNi}_{1.5} \mathrm{Ti}_{y}$ high entropy alloys. Acta Materialia, 2011, 59(16): 6308-6317.

[10] JIANG S C, HU T, GILD J, et al. A new class of high-entropy perovskite oxides. Scripta Materialia, 2018, 142: 116-120.

[11] TSAI M H. Physical properties of high entropy alloys. Entropy, 
2013, 15: 5338-5345.

[12] 顾俊峰, 邹冀, 张帆, 等. 高熵陶瓷材料研究进展. 中国材料进 展, 2019, 38(9): 855-865.

[13] YEH J W. Recent progress in high-entropy alloys. Annales De Chimie-Science des Materiaux, 2006, 31: 633-648.

[14] MIRACLE D B. High-entropy alloys: a current evaluation of founding ideas and core effects and exploring "nonlinear alloys". JOM, 2017, 69(11): 2130-2136.

[15] ROST C M, SACHET E, BORMAN T, et al. Entropy-stabilized oxides. Nature Communications, 2015, 6(1): 8485.

[16] CHELlALI M R, SARKAR A, NANDAM S H, et al. On the homogeneity of high entropy oxides: an investigation at the atomic scale. Scripta Materialia, 2019, 166: 58-63.

[17] DJENADIC R, SARKAR A, CLEMENS O, et al. Multicomponent equiatomic rare earth oxides. Materials Research Letters, 2017, 5(2): 102-109.

[18] DUPUY A D, WANG X, SCHOENUNG J M. Entropic phase transformation in nanocrystalline high entropy oxides. Materials Research Letters, 2019, 7(2): 60-67.

[19] GILD J, ZHANG Y Y, HARRINGTON T, et al. High-entropy metal diborides: a new class of high-entropy materials and a new type of ultrahigh temperature ceramics. Scientific Reports, 2016, 6: 37946.

[20] YAN X L, CONSTANTIN L, LU Y F, et al. $\left(\mathrm{Hf}_{0.2} \mathrm{Zr}_{0.2} \mathrm{Ta}_{0.2} \mathrm{Nb}_{0.2} \mathrm{Ti}_{0.2}\right) \mathrm{C}$ high-entropy ceramics with low thermal conductivity. Journal of the American Ceramic Society, 2018, 101(10): 4486-4491.

[21] CHEN H, XIANG H M, DAI F Z, et al. High porosity and low thermal conductivity high entropy $\left(\mathrm{Zr}_{0.2} \mathrm{Hf}_{0.2} \mathrm{Ti}_{0.2} \mathrm{Nb}_{0.2} \mathrm{Ta}_{0.2}\right) \mathrm{C}$. Journal of Materials Science \& Technology, 2019, 35(8): 1700-1705.

[22] CASTlE E, CSANADI T, GRASSO S, et al. Processing and properties of high-entropy ultra-high temperature carbides. Scientific Reports, 2018, 8(1): 8609.

[23] SARKER P, HARRINGTON T, TOHER C, et al. High-entropy high-hardness metal carbides discovered by entropy descriptors. Nature Communications, 2018, 9(1): 4980.

[24] YE B L, WEN T Q, NGUYEN M C, et al. First-principles study, fabrication and characterization of $\left(\mathrm{Zr}_{0.25} \mathrm{Nb}_{0.25} \mathrm{Ti}_{0.25} \mathrm{~V}_{0.25}\right) \mathrm{C}$ highentropy ceramics. Acta Materialia, 2019, 170: 15-23.

[25] HARRINGTON T J, GILD J, SARKER P, et al. Phase stability and mechanical properties of novel high entropy transition metal carbides. Acta Materialia, 2019, 166: 271-280.

[26] YE B L, WEN T Q, HUANG K H, et al. First-principles study, fabrication, and characterization of $\left(\mathrm{Hf}_{0.2} \mathrm{Zr}_{0.2} \mathrm{Ta}_{0.2} \mathrm{Nb}_{0.2} \mathrm{Ti}_{0.2}\right) \mathrm{C}$ highentropy ceramic. Journal of the American Ceramic Society, 2019, 102(7): 4344-4352.

[27] WANG K, CHEN L, XU C G, et al. Microstructure and mechanical properties of (TiZrNbTaMo)C high-entropy ceramic. Journal of Materials Science \& Technology, 2020, 39: 99-105.

[28] ZHANG W, CHEN L, XU C G, et al. Densification, microstructure and mechanical properties of multicomponent (TiZrHfNbTaMo)C ceramic prepared by pressureless sintering. Journal of Materials Science \& Technology, 2021, 72: 23-28.

[29] JIN T, SANG X H, UNOCIC R R, et al. Mechanochemicalassisted synthesis of high-entropy metal nitride via a soft urea strategy. Advanced Materials, 2018, 30(23): 1707512.

[30] GILD J, BRAUN J, KAUFMANN K, et al. A high-entropy silicide: $\left(\mathrm{Mo}_{0.2} \mathrm{Nb}_{0.2} \mathrm{Ta}_{0.2} \mathrm{Ti}_{0.2} \mathrm{~W}_{0.2}\right) \mathrm{Si}_{2}$. Journal of Materiomics, 2019, 5(3): 337-343.

[31] ZHAO Z F, XIANG H M, DAI F Z, et al. (TiZrHf) $\mathrm{P}_{2} \mathrm{O}_{7}$ : an equimolar multicomponent or high entropy ceramic with good thermal stability and low thermal conductivity. Journal of Materials Science \& Technology, 2019, 35(10): 2227-2231.

[32] LIU Y C, JIA D C, ZHOU Y, et al. $\mathrm{Zn}_{0.1} \mathrm{Ca}_{0.1} \mathrm{Sr}_{0.4} \mathrm{Ba}_{0.4} \mathrm{ZrO}_{3}$ : a non-equimolar multicomponent perovskite ceramic with low thermal conductivity. Journal of the European Ceramic Society, 2020, 40: 6272-6277.

[33] ZHU D M. Advanced Environmental Barrier Coatings for $\mathrm{SiC} / \mathrm{SiC}$ Ceramic Matrix Composite Turbine Components. Engineered
Ceramics: Current Status and Future Prospects, Hoboken, New Jersey: John Wiley \& Sons, Inc, 2016.

[34] LEE K N, FOX D S, BANSAL N P. Rare earth silicate environmental barrier coatings for $\mathrm{SiC} / \mathrm{SiC}$ composites and $\mathrm{Si}_{3} \mathrm{~N}_{4}$ ceramics. Journal of the European Ceramic Society, 2005, 25(10): 1705-1715.

[35] 田志林. 几种稀土硅酸盐陶瓷的预测、制备和性能研究. 北京: 中国科学院大学博士学位论文, 2016.

[36] LUO Y X, SUN L C, WANG J M, et al. Tunable thermal properties in yttrium silicates switched by anharmonicity of low-frequency phonons. Journal of the European Ceramic Society, 2018, 38: 2043-2052.

[37] POERSCHKE D L, HASS D D, EUSTIS S, et al. Stability and CMAS resistance of ytterbium-silicate/hafnate $\mathrm{EBCs} / \mathrm{TBC}$ for $\mathrm{SiC}$ composites. Journal of the American Ceramic Society, 2015, 98(1): 278-286.

[38] DONG Y, REN K, LU Y H, et al. High-entropy environmental barrier coating for the ceramic matrix composites. Journal of the European Ceramic Society, 2019, 39: 2574-2579.

[39] CHEN H, XIANG H M, DAI F Z, et al. High entropy $\left(\mathrm{Yb}_{0.25} \mathrm{Y}_{0.25} \mathrm{Lu}_{0.25} \mathrm{Er}_{0.25}\right)_{2} \mathrm{SiO}_{5}$ with strong anisotropy in thermal expansion. Journal of Materials Science \& Technology, 2020, 36: 134-139.

[40] REN X M, TIAN Z L, ZHANG J, et al. Equiatomic quaternary $\left(\mathrm{Y}_{1 / 4} \mathrm{Ho}_{1 / 4} \mathrm{Er}_{1 / 4} \mathrm{Yb}_{1 / 4}\right)_{2} \mathrm{SiO}_{5}$ silicate: a perspective multifunctional thermal and environmental barrier coating material. Scripta Materialia, 2019, 168: 47-50.

[41] RIDLEY M, GASKINS J, HOPKINS P, et al. Tailoring thermal properties of multi-component rare earth monosilicates. Acta Materialia, 2020, 195: 698-707.

[42] TURCER L R, SENGUPTA A, PADTURE N P. Low thermal conductivity in high-entropy rare-earth pyrosilicate solid-solutions for thermal environmental barrier coatings. Scripta Materialia, 2021, 191: 40-45.

[43] POERSCHKE D L, JACKSON R W, LEVI C G. Silicate deposit degradation of engineered coatings in gas turbines: progress toward models and materials solutions. Annual Review of Materials Research, 2017, 47: 297-330.

[44] LIU J, ZHANG L T, LIU Q M, et al. Calcium-magnesiumaluminosilicate corrosion behaviors of rare-earth disilicates at $1400{ }^{\circ} \mathrm{C}$. Journal of the European Ceramic Society, 2013, 33: 3419-3428.

[45] TIAN Z L, REN X M, LEI Y M, et al. Corrosion of $\mathrm{RE}_{2} \mathrm{Si}_{2} \mathrm{O}_{7}$ $(\mathrm{RE}=\mathrm{Y}, \mathrm{Yb}$, and $\mathrm{Lu})$ environmental barrier coating materials by molten calcium-magnesium-alumino-silicate glass at high temperatures. Journal of the European Ceramic Society, 2019, 39: 4245-4254.

[46] TURCER L R, KRAUSE A R, GARCES H F, et al. Environmental-barrier coating ceramics for resistance against attack by molten calcia-magnesia-aluminosilicate (CMAS) glass: Part I, $\mathrm{YAlO}_{3}$ and $\gamma-\mathrm{Y}_{2} \mathrm{Si}_{2} \mathrm{O}_{7}$. Journal of the European Ceramic Society, 2018, 38: 3905-3913.

[47] TURCER L R, KRAUSE A R, GARCES H F, et al. Environmental-barrier coating ceramics for resistance against attack by molten calcia-magnesia-aluminosilicate (CMAS) glass: Part II, $\beta-\mathrm{Yb}_{2} \mathrm{Si}_{2} \mathrm{O}_{7}$ and $\beta-\mathrm{Sc}_{2} \mathrm{Si}_{2} \mathrm{O}_{7}$. Journal of the European Ceramic Society, 2018, 38: 3914-3924.

[48] SUN L C, LUO Y X, TIAN Z L, et al. High temperature corrosion of $\left(\mathrm{Er}_{0.25} \mathrm{Tm}_{0.25} \mathrm{Yb}_{0.25} \mathrm{Lu}_{0.25}\right)_{2} \mathrm{Si}_{2} \mathrm{O}_{7}$ environmental barrier coating material subjected to water vapor and molten calcium-magnesiumaluminosilicate (CMAS). Corrosion Science, 2020, 175: 108881.

[49] FELSCHE J. The Crystal Chemistry of the Rare-earth Silicates. Rare Earths. Structure and Bonding, Vol 13. Berlin, Heidelberg: Springer, 1973.

[50] SUN L C, LUO Y X, REN X M, et al. A multicomponent $\gamma$-type $\left(\mathrm{Gd}_{1 / 6} \mathrm{~Tb}_{1 / 6} \mathrm{Dy}_{1 / 6} \mathrm{Tm}_{1 / 6} \mathrm{Yb}_{1 / 6} \mathrm{Lu}_{1 / 6}\right)_{2} \mathrm{Si}_{2} \mathrm{O}_{7}$ disilicate with outstanding thermal stability. Materials Research Letters, 2020, 8(11): 424-430. 